EDITORIAL

\title{
Exercise medicine in men with prostate cancer: breaking barriers to increase participation
}

(C) The Author(s), under exclusive licence to Springer Nature Limited 2021

Prostate Cancer and Prostatic Diseases (2021) 24:942-943; https:// doi.org/10.1038/s41391-021-00406-4

Exercise intervention studies in men with prostate cancer have shown well documented improvements in objective and selfreported outcomes [1] with higher levels of physical activity also associated with reduced prostate cancer specific and overall mortality [2]. Recently, exercise has been proposed as a medicine in the setting of oncology with initial recommendations presented to guide clinicians in directing and referring patients to undertake exercise programs during/following treatments [3].

However, despite supporting data from several clinical trials, the majority of men with prostate cancer do not undertake exercise programs or are regularly physically active. For example, we previously reported data of a population-based cohort of metropolitan and rural Australian men with prostate cancer on adherence to exercise-oncology recommendations including aerobic and resistance exercise modes [4]. Only $~ 12 \%$ of men reported undertaking recommended exercise levels $(150 \mathrm{~min}$ of moderate intensity or $75 \mathrm{~min}$ of strenuous exercise per week and twice weekly resistance exercise) with $\sim 47 \%$ being completely inactive [4]. As expected, lack of physical activity also contributed to poorer quality of life. Given the low use of exercise in the clinical setting when treating men with prostate cancer and overall low levels of physical activity of these men, identifying barriers, facilitators and preferences to increase exercise participation and referrals to exercise programs that can improve outcomes in men with prostate cancer should be an important priority.

In this issue of Prostate Cancer and Prostatic Diseases, Sattar et al. [5]. presented findings from a comprehensive scoping review examining facilitators, preferences, and barriers to exercise and physical activity in men with prostate cancer. The authors reported 46 studies presenting quantitative and qualitative results on these outcomes and are to be commended for this work. For the purpose of their review, physical activity was defined as any bodily movement produced by skeletal muscles that results in energy expenditure, with the inclusion of structured planned exercise as well as sport-based activities with the intention to capture all studies. From a treatment perspective, studies involved men with prostate cancer who had received a prostatectomy or were undergoing and/or had received androgen deprivation therapy (ADT) with or without radiation therapy.

The authors report that the main preference among men with prostate cancer was to exercise in a structured group environment. Our clinical and research experience is that group exercise is highly engaging for men with prostate cancer and facilitates the uptake of exercise programs and enhances long-term adherence to exercise. Structured group exercise likely facilitates the interaction with peers and provides as a supportive group environment. However, in studies including patients with other cancers, exercise preferences have differed across reports in terms of patient preferences in relation to undertaking exercise in a group format vs. alone or other components of exercise programming including different modes of exercise, location of the exercise program and supervision. For example, in a early study in this field which included patients with prostate, breast, colorectal and lung cancer, patients reported a preference to exercise alone [6]. Clearly, an approach that considers individual preferences must be used when planning the prescription of exercise programs to ensure long-term adherence and compliance. However, in addition to patient preferences, it is important to inform patients that specific exercise programs may be required to achieve defined exercise goals (e.g., reduce body fat, counteract loss of muscle mass following/during ADT). Consequently, a balance between patient preference and specificity of exercise to improve patient outcomes should be recommended.

A common barrier to participation in exercise programs reported among cancer patients was treatment-related sideeffects. Again, it is important for patients to be well-informed on the benefits of exercise in reducing specific treatment-related adverse effects. For example, in the case of fatigue during ADT, men with prostate cancer with the highest levels of fatigue and lowest vitality have reported the greatest benefits in a previous yearlong study suggesting an important exercise response in those with more symptoms at baseline [7]. Another exercise barrier reported consistently across many studies is lack of time. If exercise is to be viewed as part of the treatment plan with clear referral pathways to an exercise specialists then this may facilitate exercise adoption and participation. For example, in a large study of 859 men with prostate cancer where exercise was offered as part of the treatment plan in patients receiving leuprorelin acetate, a very high compliance rate of $92 \%$ was reported [8]. In our view, having exercise as part of the treatment plan is a major facilitator to improve participation.

The authors also reported on masculinity as a prominent theme noted from patient perspectives. In our experience, a key care consideration in men with prostate cancer is the adoption of a men-centered approach that considers masculinities and genderspecific care preferences. Physical strength, fitness, and an actionoriented coping approach are described by men as aspects of masculinity important in their prostate cancer experience [9]. In this sense, if appropriately framed and presented to men, exercise medicine has a unique capacity to overcome barriers to helpseeking by simply being more socially or personally acceptable. In brief, exercise medicine provides the opportunity to work synergistically with masculine values to assist in recovery from a psychological and masculine identity perspective, thereby improving patient outcomes.

Last, exercise medicine has the potential to support or enhance personal agency (defined as the capacity of an individual to initiate, execute and manage their actions in response to the awareness and ownership of health-related needs), an essential core component of quality survivorship care [10]. It is notable that in a recent review of survivorship interventions strong evidence 
was found for the effectiveness of both exercise medicine and psychosocial interventions to improve patient outcomes [11]. Survivorship care that incorporates these domains of care is essential.

In all, it is now time to break exercise barriers and promote exercise facilitators so we can have more men with prostate cancer walking, running, cycling, lifting weights, and benefiting from exercise medicine!

Daniel A. Galvão $\mathbb{D}^{1,2 \bowtie}$ and Suzanne K. Chambers ${ }^{1,3,4}$ ${ }^{1}$ Exercise Medicine Research Institute, Edith Cowan University, Joondalup, WA, Australia. ${ }^{2}$ School of Medical and Health Sciences, Edith Cowan University, Joondalup, WA, Australia. ${ }^{3}$ Faculty of Health

Sciences, Australian Catholic University, Brisbane, QLD, Australia.

${ }^{4}$ Faculty of Health, University of Technology Sydney, Ultimo, NSW, Australia. ${ }^{\bowtie}$ email: d.galvao@ecu.edu.au

\section{REFERENCES}

1. Galvao DA, Taaffe DR, Spry N, Joseph D, Newton RU. Combined resistance and aerobic exercise program reverses muscle loss in men undergoing androgen suppression therapy for prostate cancer without bone metastases: a randomized controlled trial. J Clin Oncol. 2010;28:340-7.

2. Kenfield SA, Stampfer MJ, Giovannucci E, Chan JM. Physical activity and survival after prostate cancer diagnosis in the health professionals follow-up study. J Clin Oncol. 2011;29:726-32.

3. Schmitz KH, Campbell AM, Stuiver MM, Pinto BM, Schwartz AL, Morris GS, et al. Exercise is medicine in oncology: engaging clinicians to help patients move through cancer. CA Cancer J Clin. 2019;69:46884.

4. Galvao DA, Newton RU, Gardiner RA, Girgis A, Lepore SJ, Stiller A, et al. Compliance to exercise-oncology guidelines in prostate cancer survivors and associations with psychological distress, unmet supportive care needs, and quality of life. Psycho-Oncol. 2015;24:1241-49.
5. Sattar S, Haase KR, Bradley C. Barriers and facilitators related to undertaking physical activities among men with prostate cancer: a scoping review. Prostate Cancer Prostatic Dis. (2021) In Press.

6. Jones LW, Courneya KS. Exercise counseling and programming preferences of cancer survivors. Cancer Pr. 2002;10:208-15.

7. Taaffe DR, Newton RU, Spry N, Joseph D, Chambers SK, Gardiner RA, et al. Effects of different exercise modalities on fatigue in prostate cancer patients undergoing androgen deprivation therapy: a year-long randomised controlled trial. Eur Urol. 2017;72:293-99.

8. Beydoun N, Bucci JA, Chin YS, Spry N, Newton R, Galvão DA. Prospective study of exercise intervention in prostate cancer patients on androgen deprivation therapy. J Med Imaging Radiat Oncol. 2014;58:369-76.

9. Chambers SK, Hyde MK, Oliffe JL, Lowe A, Wootten AC, Dunn J. Measuring masculinity in the context of chronic disease. Psychology of men \& masculinity. US: Educational Publishing Foundation; 2016. p. 228-42.

10. Dunn J, Green A, Ralph N, Newton RU, Kneebone A, Frydenberg M, et al. Prostate cancer survivorship essentials framework: guidelines for practitioners. BJU Int. 2020 https://doi.org/10.1111/bju.15159 [published Online First:2020/07/07].

11. Crawford-Williams F, March S, Goodwin BC, Ralph N, Galvão DA, Newton RU, et al. Interventions for prostate cancer survivorship: a systematic review of reviews. Psycho-Oncol. 2018;27:2339-48.

\section{CONFLICT OF INTEREST}

The authors declare no competing interests.

\section{ADDITIONAL INFORMATION}

Correspondence and requests for materials should be addressed to D.A.Gão.

Reprints and permission information is available at http://www.nature.com/ reprints

Publisher's note Springer Nature remains neutral with regard to jurisdictional claims in published maps and institutional affiliations. 